\title{
Study of Existence of Almost Automorphic Solutions to Some Singular Systems of Differential Equations
}

\author{
Gradi KAMINGU LUBWELE* \\ Candidate Assistant, Faculty of Sciences, Department of Mathematics and Computer Science, University of \\ Kinshasa, DR Congo. \\ *Corresponding Author: Gradi KAMINGU LUBWELE, Candidate Assistant, Faculty of Sciences, \\ Department of Mathematics and Computer Science, University of Kinshasa, DR Congo.
}

Abstract: This paper deals with the existence of almost automorphic solutions to a nonhomogeneous singular system of differential equations

$$
E u^{\prime}(t)+F u(t)=g(t), \text { for all } t \in \mathbb{R},
$$

where $E, F$ are $m \times m$-square matrices with complex entries such that: $\operatorname{det}(E)=0, \operatorname{det}(F) f \neq 0$ and $g$ is an almost automorphic function. To do this, we make use of various tools and techniques from functional analysis. To illustrate our abstract results, we discuss some examples.

Keywords: Almost automorphic functions, Singular system, Differential Equations, Almost periodic functions, Existence of Solutions.

\section{INTRODUCTION}

Nearly a century ago, the concept of periodicity was in full swing. It was not until 1925 that the work of the Danish mathematician Harald August Bohr gave rise to the concept of almost periodicity. This concept comes to generalize the notion of periodicity. At first glance, a periodic function is a function that repeats its values at regular intervals or periods. This paper begins with the introduction of periodic, almost periodic and almost automorphic functions. Several classical examples show that the almost periodic functions are a generalization of periodic functions and that the almost automorphic functions are a generalization of almost periodic functions.

Several interesting works deal with the concept of almost automorphy, namely the book by Diagana [8] and that of N'Guérékata [12] and the series of papers by Shen and Yi [14].

In this Thesis, we study the following system of singular differential equations,

$E u^{\mathrm{J}}(t)+F u(t)=g(t)$, for all $t \in \mathrm{R}$,

where $E, F$ are $m m$ square matrices with complex entries and the function $g(t)$ is an almost automorphic function. In this work, we suppose that $E$ is $\operatorname{singular}(\operatorname{det}(E)=0)$ and that $F$ is invertible.

In view of the problem, in this paper, we shall examine the following point: under what conditions does (1.1) have an almost automorphic (mild, classical) solution, possibly unique?

Several methods and techniques are used for the resolution to Eq. (1.1). In our case, we use a lot of the techniques developed in the paper of Diagana and Mbaye [9].

It should be noted that the concept of almost automorphy was initiated by Bochner (1955). Most of the definitions and concepts used in this section come from the books by Diagana [8] and that of N'Guérékata [12]. For more on these classes of functions and related issues, we refer the reader to the above-mentioned books.

In the sequel, the notation $(X$, . I) \$tands for a Banach space. Similarly, $B(\mathrm{R}, X), C(\mathrm{R}, X)$ and $B C(\mathrm{R}, X)$ which stand respectively for the family of bounded functions from $\mathrm{R}$ to $X$, 
the family of continuous functions from $\mathrm{R}$ to $X$ and the Banach space of bounded continuous functions from $X$ to $Y$, equipped with corresponding sup norm defined by $\|f\|_{\infty}:=\sup \|f(t)\|$.

In doing so, this paper is organized in six sectipions, the first being the present introduction. In the second section, we define the concept of almost automorphic function and give its different properties. In the third section, we study the conditions under which we obtain the existence of automorphic solutions to Eq. (1.1). In the fourth section, we use an example to illustrate our results. Finally, in conclusion, we discuss future work and open problems.

\subsection{Periodic and Almost Periodic Functions}

In this subsection we recall the concepts of periodicity and almost periodicity, particular cases of almost automorphy.

Définition 1.1.1. If $f: \mathbb{R} \longrightarrow X$ is a function, we denote the translate of $f$ by $s \in \mathbb{R}$ the function defined by

$$
\mathcal{R}_{s} f(t):=f(t+s), \forall t \in \mathbb{R} \text {. }
$$

Définition 1.1.2 (Periodic Function). A function $f \in C(\mathbb{R}, X)$ is said to be periodic, if there exists $\tau \in \mathbb{R}$, such that:

$$
\mathcal{R}_{\tau} f(t)=f(t), \quad \text { for all } t \in \mathbb{R} .
$$

In this case, $\tau$ is called a period of the function $f$.

Exemple 1.1.1. Functions such as $f(t)=\frac{1}{3} \sin (2 t), h(\theta)=1-\cos (\theta), g(x)=-2 \cos (4 x+5)$ and cis $(t)=\cos (t)+i \sin (t)$ are periodic. It is easy to verify that the number $\tau$ exists for each of these three functions.

The graphs of the periodic function Sin (in blue) and Cos (in red) are given in Fig 1 .

Exemple 1.1.2. Let us take the function defined as follows:

$$
f(t)=\sin (\sqrt{2} t)+\sqrt{3} \cos t .
$$

The function in Eq.(1.1.2), although continuous, is not periodic.

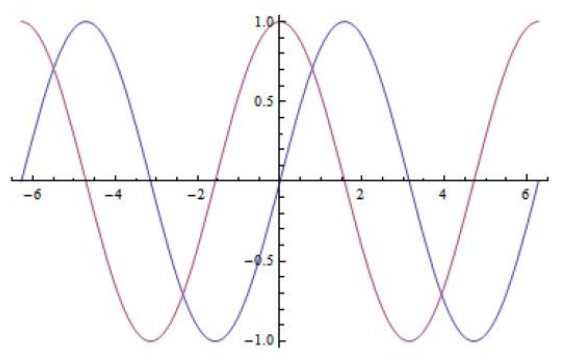

Figure1. Graphs of the $2 \pi$ periodic functions Cosine and Sine

Définition 1.1.3 ([3], [4]). Let $f \in C(\mathbb{R}, X)$. We say that $f$ is (Bohr) almost periodic function if for each $\epsilon>0$, there exists $l(\epsilon)>0$, such that $\forall a \in I, \exists \tau \in[a, a+l]$ with the property:

$$
\sup _{t \in \mathbb{R}}\left\|\mathcal{R}_{\tau} f(t)-f(t)\right\|_{\infty}<\epsilon .
$$

$\tau$ is then called an $\epsilon$-period or $\epsilon$-translation.

The collection (space) of almost periodic functions $f: \mathbb{R} \longrightarrow X$ will be denoted $A P(\mathbb{R}, X)$ or simply $A P(X)$.

Let us recall that a trigonometric polynomial is any function of the following form:

$$
\begin{aligned}
T_{n}(t) & =\sum_{k=0}^{n} a_{k} e^{i \lambda_{k} t}, \\
& =\sum_{k=0}^{n} a_{k}\left(\cos \left(\lambda_{k} t\right)+i \sin \left(\lambda_{k} t\right)\right),
\end{aligned}
$$

where $\lambda_{k} \in \mathbb{R} ; a_{k} \in \mathbb{C}$.

Définition 1.1 .4 ([7]). A function $f \in A P(X)$ if and only if there exists a trigonometric polynomial $T_{n}$ such that:

$$
\lim _{n \longrightarrow \infty}\left\|f(t)-T_{n}(t)\right\|=0 .
$$


Définition 1.1.5 (Bochner [2]). Let $f \in B C(\mathbb{R}, X)$. We say that $f$ is a (Bochner) almost periodic function if any sequence $\left(s_{n}^{\prime}\right)_{n \in \mathbb{N}}$ of real numbers, there exists a subsequence $\left(s_{n}\right)_{n \in \mathbb{N}}$ such that $f\left(t+s_{n}\right)$ converges uniformly in $t \in \mathbb{R}$, i.e., $\exists g \in B C(\mathbb{R}, X)$ such that:

$$
\lim _{n \rightarrow \infty} \sup _{t \in \mathbb{R}}\left\|f\left(t+s_{n}\right)-g(t)\right\|=0 .
$$

Théorème 1.1.1 ([7]). A function $f \in B C(\mathbb{R}, X)$ is Bohr almost periodic if and only if it is Bochner almost periodic.

Rémarque 1.1.1. There exist almost periodic functions that are not periodic functions.

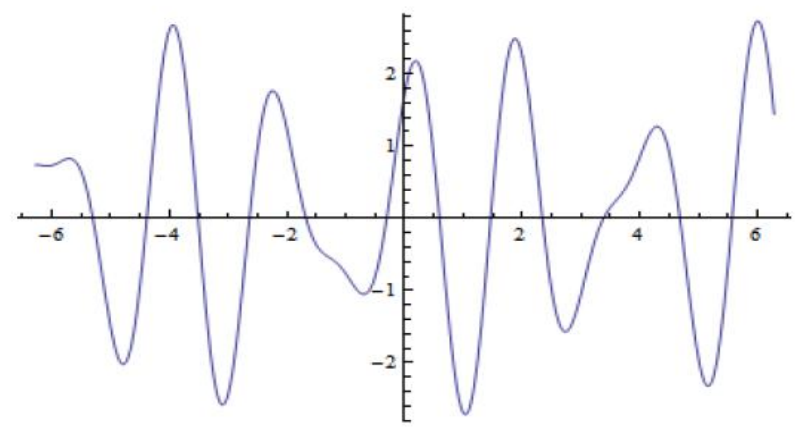

Figure2. Graph of the fonction 1.1.2

Proof. In what follows we give an example of an almost periodic function which is not periodic. So, set $f(t)=e^{i t}+e^{i \pi t}$ and suppose that there exists a number $\tau \in \mathbb{R}$, such that $\mathcal{R}_{s} f(t)=f(t), \forall t \in \mathbb{R}$. Then we have what follows:

$$
\mathcal{R}_{s} f(t):=f(\tau+t)=f(t) .
$$

Therefore,

$$
\begin{gathered}
e^{i(\tau+t)}+e^{i \pi(\tau+t)}=e^{i t}+e^{i \pi t}, \\
e^{i(\tau+t)}-e^{i t}+e^{i \pi(\tau+t)}-e^{i \pi t}=0, \\
\left(e^{i \tau}-1\right) e^{i t}+\left(e^{i \pi \tau}-1\right) e^{i \pi t}=0 .
\end{gathered}
$$

Since $e^{i t}$ and $e^{i \pi t}$ are linear independent, then $e^{i \tau}-1=0$ and $e^{i \pi \tau}-1=0$ which imply that, $e^{i \tau}=1, e^{i \pi \tau}=1$. Therefore, $\tau$ must satisfy the conditions that $e^{i \tau}=e^{i \pi \tau}$.

Then we have $\tau=2 k \pi, \pi \tau=2 h \pi$ where $k$ and $h$ are integers.

This means that

$$
\left\{\begin{array}{l}
\tau=2 k \pi \\
\tau=2 h \frac{\pi}{\pi}=2 h
\end{array}\right.
$$

which is impossible. Consequently, $\mathrm{f}$ is almost periodic and not periodic.

It should be mentioned that if the convergence in Definition ?? is uniform in, $t \in \mathbb{R}$, then we get the almost periodicity of the function $f$. Thus, the concept of almost automorphy is more general than the concept of almost periodicity

Example 1.1.3. Here are the most common examples of almost automorphic functions:

- Every almost periodic function is almost automorphic function.

- $f(t)=\cos \left(\frac{1}{p(t)}\right)$, where $p(t)=2+\sin \alpha t+\sin \beta t$ with $\alpha \beta^{-1}$ is an irrational number.

- The famous example from Veech ([13]),

$$
f(t)=\frac{2+e^{i t}+e^{i t \sqrt{2}}}{\left|2+e^{i t}+e^{i t \sqrt{2}}\right|} \text { for all } t \in \mathbb{R} .
$$

The collection (space) of almost automorphic functions $f: \mathbb{R} \longrightarrow X$ will be denoted $A A(\mathbb{R}, X)$ or simply $A A(X)$.

\subsection{Properties of Almost Automorphic Functions}

In this subsection, we discuss basic properties of almost automorphic functions. 
Théorème 1.2.1. If $f \in A A(X), \alpha, \beta \in \mathbb{R}$, then:

(i) $\alpha f \in A A(X)$;

(ii) $t \longmapsto f(\alpha+t) \in A A(X)$;

(iii) $t \longmapsto f(\alpha t) \in A A(X)$, in particular $t \longmapsto f(-t) \in A A(X)$.

Proof. Let $\left(s_{n}^{\prime}\right)_{n \in \mathbb{N}}$ be a sequence of real numbers. Since $f \in A A(X)$, we can extract a subsequence $\left(s_{n}\right)_{n \in \mathbb{N}} \subset\left(s_{n}^{\prime}\right)_{n \in \mathbb{N}}$, such that:

$$
\lim _{n \longrightarrow \infty} f\left(t+s_{n}\right)=g(t)
$$

and

$$
\lim _{n \longrightarrow \infty} g\left(t-s_{n}\right)=f(t)
$$

for each $t \in \mathbb{R}$.

(i) Clearly,

$$
\begin{aligned}
\lim _{n \longrightarrow \infty} \alpha f\left(t+s_{n}\right) & =\alpha \lim _{n \longrightarrow \infty} f\left(t+s_{n}\right) \\
& =\alpha g(t)
\end{aligned}
$$

and

$$
\begin{aligned}
\lim _{n \longrightarrow \infty} \alpha g\left(t-s_{n}\right) & =\alpha \lim _{n \longrightarrow \infty} g\left(t-s_{n}\right) \\
& =\alpha f(t),
\end{aligned}
$$

for each $t \in \mathbb{R}$.

Thus $\alpha f \in A A(X)$.

And

(ii) Let us prove that $t \longmapsto f_{\alpha}:=f(\alpha+t) \in A A(X)$.

$$
\begin{aligned}
f_{\alpha}\left(t+s_{n}\right) & =f\left(\alpha+t+s_{n}\right), \text { put } \alpha+t=s, \text { then, it follows } \\
\lim _{n \rightarrow \infty} f_{\alpha}\left(t+s_{n}\right) & =\lim _{n \longrightarrow \infty} f\left(\alpha+t+s_{n}\right) \\
& =\lim _{n \rightarrow \infty} f\left(s+s_{n}\right) \\
& =g(s)=g(\alpha+t)
\end{aligned}
$$

and

$$
\begin{aligned}
g\left(t-s_{n}\right) & =f\left(\alpha+t-s_{n}\right), \text { then it follows } \\
\lim _{n \longrightarrow \infty} g\left(t-s_{n}\right) & =\lim _{n \rightarrow \infty} g\left(\alpha+t-s_{n}\right) \\
& =\lim _{n \rightarrow \infty} g\left(s-s_{n}\right) \\
& =f_{\alpha}(s)=f(\alpha+t),
\end{aligned}
$$

for $t \in \mathbb{R}$.

Hence, $t \longmapsto f(\alpha+t) \in A A(X)$.

(iii) Let us prove that $t \longmapsto f(\alpha t) \in A A(X)$.

$$
\begin{aligned}
f\left(\alpha\left(t+s_{n}\right)\right) & =f\left(\alpha t-\alpha s_{n}\right), \text { put } \alpha s_{n}=\sigma_{n}, \text { then, it follows } \\
\lim _{n \longrightarrow \infty} f\left(\alpha\left(t+s_{n}\right)\right) & =\lim _{n \rightarrow \infty} f\left(\alpha t-\sigma_{n}\right) \\
& =g(\alpha t) \\
g\left(\alpha\left(t-s_{n}\right)\right) & =g\left(\alpha t-\alpha s_{n}\right), \text { then it follows } \\
\lim _{n \rightarrow \infty} f\left(\alpha\left(t-s_{n}\right)\right) & =\lim _{n \rightarrow \infty} g\left(\alpha t-\alpha \sigma_{n}\right) \\
& =f(\alpha t),
\end{aligned}
$$

for $t \in \mathbb{R}$.

In particular if $\alpha=-1$, then $t \longmapsto f(-t) \in A A(X)$. 
Théorème 1.2.2. Let $f, g \in A A(X)$, then sum $f+g$ belongs to $A A(X)$.

Proof. Since $f \in A A(X)$, for any sequence $\left(s_{n}^{\prime \prime}\right)_{n \in \mathbb{N}}$ of real numbers, we can extract a subsequence $\left(s_{n}^{\prime}\right)_{n \in \mathbb{N}}$ of $\left(s_{n}^{\prime \prime}\right)_{n \in \mathbb{N}}$ such that

$$
\lim _{n \rightarrow \infty} f\left(t+s_{n}^{\prime}\right)=h_{1}(t)
$$

and

$$
\lim _{n \rightarrow \infty} h_{1}\left(t-s_{n}^{\prime}\right)=f(t)
$$

for each $t \in \mathbb{R}$.

Since $g \in A A(X)$, we can extract a subsequence $\left(s_{n}\right)_{n \in \mathbb{N}}$ of $\left(s_{n}^{\prime}\right)_{n \in \mathbb{N}}$ such that

$$
\lim _{n \rightarrow \infty} g\left(t+s_{n}\right)=h_{2}(t)
$$

and

$$
\lim _{n \rightarrow \infty} h_{2}\left(t-s_{n}\right)=g(t)
$$

for each $t \in \mathbb{R}$.

Let us put $h=f+g$.

Now,

$$
\begin{aligned}
& (f+g)\left(t+s_{n}\right)=f\left(t+s_{n}\right)+g\left(t+s_{n}\right), \text { then it follows } \\
& \begin{aligned}
(f+g)\left(t+s_{n}\right)= & f\left(t+s_{n}\right)+g\left(t+s_{n}\right), \text { then it follows } \\
\lim _{n \rightarrow \infty} h\left(t+s_{n}\right) & =\lim _{n \longrightarrow \infty}\left[f\left(t+s_{n}\right)+g\left(t+s_{n}\right)\right] \\
& =\lim _{n \longrightarrow \infty} f\left(t+s_{n}\right)+\lim _{n \rightarrow \infty} g\left(t+s_{n}\right) \\
& =h_{1}(t)+h_{2}(t) \\
& =\varphi(t)\left(\text { by putting } \varphi=h_{1}+h_{2}\right)
\end{aligned}
\end{aligned}
$$

and

$$
\begin{aligned}
& \varphi\left(t-s_{n}\right)=h_{1}\left(t-s_{n}\right)+h_{2}\left(t-s_{n}\right), \text { then it follows } \\
& \lim _{n \rightarrow \infty} \varphi\left(t-s_{n}\right)=\lim _{n \rightarrow \infty}\left[h_{1}\left(t-s_{n}\right)+h_{2}\left(t-s_{n}\right)\right] \\
& \lim _{n \rightarrow \infty} \varphi\left(t-s_{n}\right)=\lim _{n \rightarrow \infty} h_{1}\left(t-s_{n}\right)+\lim _{n \rightarrow \infty} h_{2}\left(t-s_{n}\right) \\
& =f(t)+g(t) .
\end{aligned}
$$

for each $t \in \mathbb{R}$, hence $h \equiv f+g \in A A(Y)$.

Théorème 1.2.3. If $f \in A A(X)$, then $f$ is a bounded, that is

$$
\sup _{t \in \mathbb{R}}\|f(t)\|=M<\infty .
$$

Proof. This proof is taken from 12

Let us prove it by contradiction. Suppose that $\sup _{t \in \mathbb{R}}\|f(t)\|=\infty$, then there exists a sequence of reals $\left(s_{n}^{\prime}\right)_{n \in \mathbb{N}}$ such that $\lim _{t \rightarrow \infty}\left\|f\left(s_{n}^{\prime}\right)\right\|=\infty$.

Since, we know by hypothesis that $f \in A A(X)$, we can then extract a subsequence $\left(s_{n}^{\prime}\right)_{n \in \mathbb{N}}$, such that $\lim _{t \rightarrow \infty} f\left(s_{n}\right)=\alpha$ exists, this implies that $\lim _{t \rightarrow \infty}\left\|f\left(s_{n}\right)\right\|=\|\alpha\|=M<\infty$, which contradicts our hypothesis.

Théorème 1.2.4, 12 . The space $\left(A A(X),\|\cdot\|_{\infty}\right)$ is a Banach space. 


\subsection{Additional Properties of Almost Automorphic Functions}

In subsection 1.2, we have listed some of the elementary properties (addition, multiplication) of almost automorphic functions. In this subsection, we study other ways to construct almost automorphic functions from a given almost automorphic function.

Théorème 1.3.1 ([12]). Let $X, Y$ be two Banach spaces such that $f: \mathbb{R} \longmapsto X$ an almost automorphic function. If $h: X \longmapsto Y$ is a continuous function, then the compositionfunction defined by

$$
(h \circ f)(t):=h(f(t)): \mathbb{R} \longmapsto Y
$$

belongs to $A A(\mathbb{R}, Y)$.

Proof. Let us take $\left(s_{n}^{\prime}\right)_{n \in \mathbb{N}}$ an arbitrary sequence of real numbers. Since $f \in A A(X)$, there exists a subsequence $\left(s_{n}\right)_{n \in \mathbb{N}} \subset\left(s_{n}^{\prime}\right)_{n \in \mathbb{N}}$ such that $g(t):=\lim _{n \rightarrow \infty} f\left(t+s_{n}\right)$ and $f(t)=\lim _{n \rightarrow \infty} g\left(t-s_{n}\right)$, for all $t \in \mathbb{R}$.

Let us consider:

$$
\begin{aligned}
(h \circ f)\left(t+s_{n}\right) & =h\left(f\left(t+s_{n}\right)\right), \text { Since } h \text { is continuous, then } \\
\lim _{n \rightarrow \infty}(h \circ f)\left(t+s_{n}\right) & =\lim _{n \rightarrow \infty} h\left(f\left(t+s_{n}\right)\right) \\
& =h\left(\lim _{n \rightarrow \infty} f\left(t+s_{n}\right)\right) \\
& =h(g(t)) \\
& :=(h \circ g)(t),
\end{aligned}
$$

and similarly, let us consider:

$$
\begin{aligned}
(h \circ g)\left(t-s_{n}\right) & =h\left(g\left(t-s_{n}\right)\right), \text { Since } h \text { is continuous, then } \\
\lim _{n \rightarrow \infty}(h \circ g)\left(t-s_{n}\right) & =\lim _{n \rightarrow \infty} h\left(g\left(t-s_{n}\right)\right) \\
& =h\left(\lim _{n \rightarrow \infty} g\left(t-s_{n}\right)\right) \\
& =h(f(t)) \\
& :=(h \circ f)(t)
\end{aligned}
$$

for each $t \in \mathbb{R}$, hence $(h \circ f) \in A A(Y)$.

Lemme 1.3.1 ([12]). Let $\left(f_{n}\right)_{n \in \mathbb{N}}$ be a sequence of almost automorphic functions such that there exists a function $f \in C(\mathbb{R}, X)$ with $\left\|f_{n}-f\right\|_{\infty} \longrightarrow 0$, as $n \longrightarrow \infty$ then $f \in A A(X)$.

Théorème 1.3.2. Let $f \in A A(X)$ and and suppose that its derivative $f^{\prime}$ exists and is uniformly continuous on $\mathbb{R}$. Then $f^{\prime} \in A A(X)$.

Proof. By assumption, $\mathrm{f}$ is almost automorphic. Suppose that $f^{\prime}$ a uniformely continuous, i.e. for every every real number $\varepsilon>0$ there exists $\delta>0$ such that for every $t_{1}, t_{2} \in X$ with $\left|t_{1}-t_{2}\right|<\delta$, we have that $\left\|f\left(t_{1}\right)-f\left(t_{2}\right)\right\|$.

Take an arbitrary $t \in \mathbb{R}$ and $\delta \geq \frac{1}{n}$, we get:

$$
n \int_{0}^{\frac{1}{n}}\left(f^{\prime}(t+s)-f^{\prime}(t)\right) \mathrm{d} s=n\left(f\left(t+\frac{1}{n}\right)-f(t)\right)-f^{\prime}(t) .
$$

Then it follows:

$$
\left\|n\left(f\left(t+\frac{1}{n}\right)-f(t)\right)-f^{\prime}(t)\right\| \leq n \int_{0}^{\frac{1}{n}}\left\|f^{\prime}(t+s)-f^{\prime}(t)\right\| \mathrm{d} s
$$

We have $|t+s-t|=s<\frac{1}{n} \leq \delta$.

Put $\left(f_{n}\right)=n\left(f\left(t+\frac{1}{n}\right)-f(t)\right)-f^{\prime}(t)$.

$\forall \delta>\frac{1}{n}, \forall n>E\left(\frac{1}{\delta}\right)+1$, where $E(x)$ is the integer part of $x$, i.e. is the biggest integer that is less or equal than $x$.

We then obtain $\left\|f_{n}-f^{\prime}(t)\right\| \leq \varepsilon$, that implies that $\lim _{n \rightarrow} f_{n}(t)=f^{\prime}(t)$.

So, $f_{n}$ is a sequence of almost automorphic function, by using Lemma 1.3 .1 we conclude that $f^{\prime}$ is also almost automorphic.

Théorème 1.3.3 (Bugajewski-Diagana [5], Theorem 4.5 in [8]). . Let $f \in A A(X)$. If $h \in L^{1}(\mathbb{R})$, where $L^{1}(\mathbb{R})$ is a vector space of classes of functions once integrable in the sense of Lebesgue in $(\mathbb{R})$. Then the convolution product defined by

$$
(f \star h)(t):=\int_{-\infty}^{\infty} f(\sigma) h(t-\sigma) \mathrm{d} \sigma
$$


belongs to $A A(X)$.

Proof. Since $f$ is continuous and $h \in L^{1}(\mathbb{R})$, it is not hard to see that $t \longmapsto(f \star h)(t)$ is continuous.

Let us take $\left(s_{n}^{\prime}\right)_{n \in \mathbb{N}}$ an arbitrary sequence of real numbers. Since $f \in A A(X)$, there exists a subsequence $\left(s_{n}\right)_{n \in \mathbb{N}} \subset\left(s_{n}^{\prime}\right)_{n \in \mathbb{N}}$ such that $g(t-\sigma):=\lim _{n \longrightarrow \infty} f\left(t-\sigma+s_{n}\right)$ and $f(t-\sigma)=\lim _{n \rightarrow \infty} g\left(t-\sigma-s_{n}\right)$, for all $t, \sigma \in \mathbb{R}$.

Let us consider

$$
(f \star h)\left(t+s_{n}\right)=\int_{-\infty}^{\infty} f\left(t-\sigma+s_{n}\right) h(\sigma) \mathrm{d} \sigma \text { for all } t \in \mathbb{R} .
$$

Clearly $\left\|f\left(t-\sigma+s_{n}\right) h(\sigma)\right\| \leq\|f\|_{\infty}|h(\sigma)|$ for each $t, \sigma \in \mathbb{R}$.

$$
\begin{aligned}
\lim _{n \longrightarrow \infty}(f \star h)\left(t+s_{n}\right) & =\lim _{n \longrightarrow \infty} \int_{-\infty}^{\infty} f\left(t-\sigma+s_{n}\right) h(\sigma) \mathrm{d} \sigma \\
& =\int_{-\infty}^{\infty} \lim _{n \longrightarrow \infty} f\left(t-\sigma+s_{n}\right) h(\sigma) \mathrm{d} \sigma \text { (by Lebesgue's dominated } \\
& \text { convergence theorem) } \\
& =\int_{-\infty}^{\infty} g(t-\sigma) h(\sigma) \mathrm{d} \sigma \\
& :=(g \star h)(t)
\end{aligned}
$$

and similarly, let us consider:

$$
(g \star h)\left(t-s_{n}\right)=\int_{-\infty}^{\infty} g\left(t-\sigma-s_{n}\right) h(\sigma) \mathrm{d} \sigma \text { for all } t \in \mathbb{R} .
$$

Clearly $\left\|g\left(t-\sigma-s_{n}\right) h(\sigma)\right\| \leq\|g\|_{\infty}|h(\sigma)|$ for each $t, \sigma \in \mathbb{R}$.

$$
\begin{aligned}
& \lim _{n \rightarrow \infty}(g \star h)\left(t-s_{n}\right)=\lim _{n \rightarrow \infty} \int_{-\infty}^{\infty} g\left(t-\sigma-s_{n}\right) h(\sigma) \mathrm{d} \sigma \\
&=\int_{-\infty}^{\infty} \lim _{n \rightarrow \infty} g\left(t-\sigma-s_{n}\right) h(\sigma) \mathrm{d} \sigma \text { (by Lebesgue's dominated } \\
& \text { convergence theorem) } \\
&=\int_{-\infty}^{\infty} f(t-\sigma) h(\sigma) \mathrm{d} \sigma \\
&:=(f \star h)(t) .
\end{aligned}
$$

for each $t \in \mathbb{R}$, hence $(f \star h) \in A A(X)$.

Théorème 1.3.4 ([15]). Let $f \in A A(\mathbb{R})$. Then the integral

$$
F(t)=\int_{0}^{t} f(s) \mathrm{d} s
$$

belongs to $A A(\mathbb{R})$ if and only if $F$ belongs to $B(\mathbb{R})$.

Proof. If we suppose that $F$ is almost automorphic, clearly $F$ is bounded.

Now, if suppose the converse, i.e. we suppose that $F$ is bounded, that is $\|F(s)\|<M<\infty$ or $\sup =M<\infty$ for $t \in \mathbb{R}$, and prove that $F$ is almost automorphic. $t \in \mathbb{R}$

Let $\left(s_{n}^{\prime}\right)_{n \in \mathbb{N}}$ be a sequence of real numbers.

Since $f \in A A(X)$, we can then extract a subsequence $\left(s_{n}\right)_{n \in \mathbb{N}}$ of $\left(s_{n}^{\prime}\right)_{n \in \mathbb{N}}$ such that:

$$
\lim _{n \rightarrow \infty} f\left(s+s_{n}\right)=g(s)
$$

and

$$
\lim _{n \rightarrow \infty} g\left(s-s_{n}\right)=f(s), \text { for all } s \in \mathbb{R} \text {. }
$$

Let us consider the integral 


$$
I=\int_{0}^{t} f\left(s+s_{n}\right) \mathrm{d} s
$$

By putting $s+s_{n}=\sigma$, we obtain then:

$$
\begin{aligned}
I & =\int_{s_{n}}^{t+s_{n}} f(\sigma) \mathrm{d} \sigma \\
& =F\left(t+s_{n}\right)-F\left(s_{n}\right),
\end{aligned}
$$

where $F$ is the primitive of $f$.

Therefore,

$$
\int_{0}^{t} f\left(s+s_{n}\right) \mathrm{d} s=F\left(t+s_{n}\right)-F\left(s_{n}\right)
$$

which yields

$$
F\left(t+s_{n}\right)=F\left(s_{n}\right)+\int_{0}^{t} f\left(s+s_{n}\right) \mathrm{d} s .
$$

By introducing the limite, as $n$ tends to infinity, we have:

$$
\begin{aligned}
\lim _{n \rightarrow \infty} F\left(t+s_{n}\right) & =\lim _{n \longrightarrow \infty} F\left(s_{n}\right)+\lim _{n \longrightarrow \infty} \int_{t_{0}}^{t} f\left(s+s_{n}\right) \mathrm{d} s \\
& =\lim _{n \longrightarrow \infty} F\left(s_{n}\right)+\int_{0}^{t} \lim _{n \longrightarrow \infty} f\left(s+s_{n}\right) \mathrm{d} s \\
& =K_{1}+\int_{0}^{t} g(s) \mathrm{d} s \text { since the limit } \lim _{n \rightarrow \infty} F\left(s_{n}\right) \text { exists and } f \in A A(\mathbb{R}) \\
& =p(t) .
\end{aligned}
$$

- Let us consider now the $p\left(t-s_{n}\right)$.

Therefore, using (1.11) and (1.12), we have: $p\left(t-s_{n}\right)=p\left(s_{n}\right)+\int_{0}^{t} g(s) \mathrm{d} s$ Now, let us compute the limit of $p\left(t-s_{n}\right)$ :

$$
\begin{aligned}
\lim _{n \rightarrow \infty} p\left(t-s_{n}\right) & =\lim _{n \rightarrow \infty} p\left(s_{n}\right)+\int_{0}^{t} \lim _{n \longrightarrow \infty} g\left(s-s_{n}\right) \mathrm{d} s \\
& =K_{2}+\int_{0}^{t} f(s) \mathrm{d} s \\
& =G(t) .
\end{aligned}
$$

Let us prove now that $K_{2}$ is null. If we should prove that, the we would establish that $F(t)$ is almost automorphic, which means:

$\lim _{m \longrightarrow \infty} \lim _{n \longrightarrow \infty}$ exists for all $t \in \mathbb{R}$.

Let us denote the operation of taking double limite by $\mathcal{A}$, i.e.

$$
\begin{aligned}
\mathcal{A} F(t) & =G(t) \text { that implies } \\
\mathcal{A} \mathcal{A} F(t)=\mathcal{A}^{2} F(t) & =\mathcal{A} G(t) \\
& =K_{2}+K_{2}+F(t) .
\end{aligned}
$$

By induction, we have:

$$
\mathcal{A}^{n} F(t)=n K_{2}+F(t)
$$

and

$$
\left|\mathcal{A}^{n} F(t)\right| \leq M, \text { for } t \in \mathbb{R} \text {. }
$$

It yields,

$$
\begin{aligned}
\left|\mathcal{A}^{n} F(t)\right| & =\left|n K_{2}+F(t)\right| \\
\left|\mathcal{A}^{n} F(t)\right| & =\left|n K_{2}\right|+|F(t)| \\
\text { So }\left|n K_{2}\right| & \leq\left|\mathcal{A}^{n} F(t)\right|+|F(t)| \\
& \leq M+M \\
& \leq 2 M .
\end{aligned}
$$

This gives contradiction if $K_{2} \neq 0$, because the left-hand side will become larger and larger as $n$ increases.

Hence $K_{2}=0$, so $\lim _{n \rightarrow \infty}=F(t)$. 
Therefore, we have just established that $\mathrm{F}$ is almost automorphic.

2. Mains Results

\subsection{Mild and Classical Solutions}

Consider the differential equation

$u^{J}(t)=A u(t)+f(t), t \in \mathrm{R}$

where $\mathrm{A}$ is an $\mathrm{n} \times \mathrm{n}$ square matrix and $\mathbf{f}: \mathrm{R} \rightarrow \mathrm{X}$ is a continuous function.

Définition 2.1.1. A continuous function $u: R \rightarrow C^{n}$ is said to be a classical solution to (2.1) if $\mathrm{u}$ is of class $\mathrm{C}^{1}$ and (2.1) holds.

Définition 2.1.2. A continuous function $u: R \rightarrow C^{n}$ is called a mild solution to (2.1) if

$$
u(t)=e^{A(t-r)} u(r)+\int_{r}^{t} e^{A(t-s)} f(s) \mathrm{d} s \quad \text { for all } t, s \in \mathbb{R}, \quad t \geq s .
$$

Rémarque 2.1.1. 1. Any classical solution u to (2.1) is a mild solution.

2. A mild solution $u$ to (2.1) is a classical solution if and only if $u$ is of class $\mathrm{C}^{1}$.

In view of the above, it is easy to see that every mild solution to Eq. (2.1) is a classical solution.

Such a mild/classical solution will be called a solution.

\subsection{Existence of Almost Automorphic Solutions}

In this subsection, we discuss the existence of almost automorphic solutions to the following singular systems of differential equations:

$$
E u^{\prime}(t)+F u(t)=g(t), \text { for all } t \in \mathbb{R},
$$

where $E, F \in M(m, \mathbb{C})$ where $\operatorname{det}(E)=0$ and $\operatorname{det}(F) \neq 0$, and $g \in A A\left(\mathbb{C}^{m}\right)$.

\subsubsection{Resolution of the problem}

Let us define the matrix $T=E F^{-1}: \mathbb{C}^{m} \longrightarrow \mathbb{C}^{m}$.

Following Favini et al. ([10]) and letting

$$
F u(t)=v(t),
$$

then we have:

$$
\begin{aligned}
F^{-1} F u(t) & =F^{-1} v(t), \text { (since } F \text { is invertible) } \\
u(t) & =F^{-1} v(t) .
\end{aligned}
$$

Taking the derivative, we have:

$$
\begin{aligned}
u^{\prime}(t) & =F^{-1} v^{\prime}(t), \\
E u^{\prime}(t) & =E F^{-1} v^{\prime}(t) .
\end{aligned}
$$

Hence

$$
E u^{\prime}(t)=T v^{\prime}(t)
$$

We reduce Eq. 2.2 to the following equation,

$$
T v^{\prime}(t)+v(t)=g(t), \text { for all } t \in \mathbb{R} .
$$

The restriction of $T$ to its range (i.e. $\mathcal{R}(T)=\mathbb{X}_{1}$ ) is invertible. Hence, we can write $T$ as the following block diagonal matrix 


$$
T=\left(\begin{array}{cc}
M^{-1} & O \\
O & O
\end{array}\right)
$$

$T$.

where $M$ and the zero matrix, are $k \times k$ square matrices on $\mathbb{X}_{1}$, with $k$ is the rank of the matrix

Since $T$ is a square matrix, then it generates a $C_{0}$-semigroup on $\mathbb{X}_{1}$.

Hence, it is possible to decompose (using the block diagonal form of $T$ ) $g$ respectively through the projection $P_{\mathrm{X}_{1}}$ onto $\mathbb{X}_{1}$ and the projection $P_{\mathrm{X}_{2}}$ onto $\mathbb{X}_{2}\left(\mathcal{N}(T)=\mathbb{X}_{2}\right)$.

$g=\left(\begin{array}{l}g_{1}:=P_{\mathbf{X}_{1}} g \\ g_{2}:=P_{\mathbf{X}_{2}} g\end{array}\right)$, that implies that:

$$
\left(\begin{array}{cc}
M^{-1} & O \\
O & O
\end{array}\right)\left(\begin{array}{l}
v_{1} \\
v_{2}
\end{array}\right)^{\prime}+\left(\begin{array}{l}
v_{1} \\
v_{2}
\end{array}\right)=\left(\begin{array}{l}
g_{1} \\
g_{2}
\end{array}\right) .
$$

We can now write Eq.(2.4) in the following form:

$$
\left\{\begin{aligned}
M^{-1} v_{1}^{\prime}(t)+v_{1}(t) & =g_{1}(t), \\
v_{2}(t) & =g_{2}(t)
\end{aligned}\right.
$$

where $v_{1}(t)$ and $v_{2}(t)$ are $m \times 1$ vectors.

We note that the system of equations in $v_{1}(t)$ in Eq.(2.5), and its mild solution $v(t)=\left(\begin{array}{l}v_{1}(t) \\ v_{2}(t)\end{array}\right)$ is given as follows:

$$
\left\{\begin{array}{l}
v_{1}(t)=e^{-M(t-\sigma)} v_{1}(\sigma)+M \int_{\sigma}^{t} e^{-M(t-s)} g_{1}(s) \mathrm{d} s \\
v_{2}(t)=g_{2}(t)
\end{array}\right.
$$

for all $t, \sigma \in \mathbb{R}$ and $t \geq \sigma$.

By multiplying the system (2.6) by the quantity $e^{-\alpha t}$, we notice that the problem is equivalent to the following system:

$$
\left\{\begin{array}{l}
e^{-\alpha t} v_{1}(t)=e^{-M(t-\alpha)(t-\sigma)}\left(e^{-\alpha t} v_{1}(\sigma)\right)+M \int_{\sigma}^{t} e^{-M(t-\alpha)(t-s)}\left(e^{-\alpha s} g_{1}(s)\right) d s, \\
e^{-\alpha t} v_{2}(t)=e^{-\alpha t} g_{2}(t)
\end{array}\right.
$$

for all $t, \sigma \in \mathbb{R}$ and $t \geq \sigma$.

As mentioned in [9], we can always choose $\alpha \geq 0$ so that $e^{-(M-\alpha) t}$ is exponentially stable, i.e. the choice of $\alpha$ must be made so that there exist $K \geq 1$ and $\omega>0$, such that:

$$
\left\|e^{-M t}\right\| \leq K e^{-\omega t} \text { for all } t \geq 0 .
$$

Théorème 2.2.1. Under previous assumptions, if $g \in A A\left(\mathbb{C}^{m}\right)$, then Eq.(2.2) has a unique almost automorphic solution.

Proof. We are going to proceed in three steps: (i) we prove existence of a solution, (ii) we prove that the solution is almost automorphic, and (iii) we prove the uniqueness of the solution.

- Existence

From Eq.(2.5), we have the following:

$$
\begin{aligned}
M^{-1} M v_{1}^{\prime}(t)+M v_{1}(t) & =M g_{1}(t), \\
v_{1}^{\prime}(t)+M v_{1}(t) & =M g_{1}(t) .
\end{aligned}
$$

Let us now multiply the equation by $e^{M t}$, we get then:

$$
e^{M t} v_{1}^{\prime}(t)+e^{M t} M v_{1}(t)=e^{M t} M g_{1}(t) .
$$

On the other hand,

$$
\left(e^{M t} v_{1}(t)\right)^{\prime}=e^{M t} v_{1}^{\prime}(t)+M e^{M t} v_{1}(t)
$$


Then, we have

$$
\begin{aligned}
\left(e^{M t} v_{1}(t)\right)^{\prime} & =e^{M t} M g_{1}(t), \\
\int_{\sigma}^{t}\left(e^{M s} v_{1}(s)\right)^{\prime} \mathrm{d} s & =\int_{\sigma}^{t} e^{M s} M g_{1}(s) \mathrm{d} s \\
\left.e^{M s} v_{1}(s)\right|_{\sigma} ^{t} & =M \int_{\sigma}^{t} e^{M s} g_{1}(s) \mathrm{d} s, \\
e^{M t} v_{1}(t)-e^{M \sigma} v_{1}(\sigma) & =M \int_{\sigma}^{t} e^{M s} g_{1}(s) \mathrm{d} s, \\
e^{M t} v_{1}(t) & =e^{M \sigma} v_{1}(\sigma)+M \int_{\sigma}^{t} e^{M s} g_{1}(s) \mathrm{d} s .
\end{aligned}
$$

Therefore,

$$
v_{1}(t)=e^{-M(t-\sigma)} v_{1}(\sigma)+M \int_{\sigma}^{t} e^{-M(t-s)} g_{1}(s) \mathrm{d} s .
$$

Almost automorphy of the solution

The solution expression of (2.5) is given by $v_{1}$ and $v_{2}$. To prove that this solution is almost automorphic, we must prove that $v_{1}$ and $v_{2}$ are almost automorphic.

Now, $v_{2}$ is clearly almost automorphic given the almost automorphy of $g_{2}$. We now have to prove that $v_{1}$ is almost automorphic.

It can be shown the only bounded mild solution to Eq.(2.5) for $v_{1}$ is given by:

$$
v_{1}(t)=M \int_{-\infty}^{t} e^{-M(t-s)} g_{1}(s) \mathrm{d} s=M G(t) .
$$

Since the projecton $P_{\mathrm{X}_{1}}$ is continuous, it follows that $g_{1}$ is an almost automorphic function on $\mathbb{X}_{1}$.

Let us prove now that, for each $r<t$ the integral $v_{1}(t)$ is almost automorphic.

Let us first consider $G(t)=\int_{-\infty}^{t} e^{-M(t-s)} g_{1}(s) \mathrm{d} s$, defined as

$$
\int_{-\infty}^{t} e^{-M(t-s)} g_{1}(s) \mathrm{d} s=\lim _{r \rightarrow-\infty} \int_{r}^{t} e^{-M(t-s)} g_{1}(s) \mathrm{d} s .
$$

We see clearly for each $r<t$ the integral $\int_{r}^{t} e^{-M(t-s)} g_{1}(s) \mathrm{d} s$ exists. Moreover, we have

$$
\left\|\int_{r}^{t} e^{-M(t-s)} g_{1}(s) \mathrm{d} s\right\| \leq \frac{K}{|\omega|}\left\|g_{1}\right\|_{\infty}, \text { for all } r<t
$$

which shows that $\int_{-\infty}^{t} e^{-M(t-s)} g_{1}(s)$ is absolutely convergent.

Thus, let $\left(s_{n}^{\prime}\right)_{n \in \mathbb{N}}$ be an arbitrary sequence of real numbers. Since $g_{1}$ an almost automorphic function, we can extract a subsequence

$\left(s_{n}\right)_{n \in \mathbb{N}} \subset\left(s_{n}^{\prime}\right)_{n \in \mathbb{N}}$ such that the following properties hold:

$$
h(t)=\lim _{n \rightarrow \infty} g_{1}\left(t+s_{n}\right)
$$

is well defined for each $t \in \mathbb{R}$ and

$$
\lim _{n \longrightarrow \infty} h\left(t-s_{n}\right)=g_{1}(t)
$$


for all $\mathrm{t} \in \mathrm{R}$.

Now consider

$$
G\left(t+s_{n}\right)=\int_{-\infty}^{t+s_{n}} e^{-M\left(t+s_{n}-s\right)} g_{1}(s) \mathrm{d} s
$$

By putting $\sigma=s-s_{n}$, we obtain:

$$
\begin{aligned}
G\left(t+s_{n}\right) & =\int_{-\infty}^{t} e^{-M(t-\sigma)} g_{1}\left(\sigma+s_{n}\right) \mathrm{d} \sigma \\
& =\int_{-\infty}^{t} e^{-M(t-\sigma)} g_{n}(\sigma) \mathrm{d} \sigma
\end{aligned}
$$

where $\left(g_{n}\right)_{n \in \mathbb{N}}$.

We have also

$$
\left\|G\left(t+s_{n}\right)\right\| \leq \frac{K}{|\omega|}\left\|g_{1}\right\|_{\infty}, \forall n \in \mathbb{N} .
$$

and by coninuity of semigroup, $e^{-M(t-\sigma)} g_{n}(\sigma) \longrightarrow e^{-M(t-\sigma)} h(\sigma)$, as $n \longrightarrow \infty$ for each $\sigma \in \mathbb{R}$ and any $t \geq \sigma$.

$$
\lim _{n \rightarrow \infty} G\left(t+s_{n}\right)=\lim _{n \rightarrow \infty} \int_{-\infty}^{t} e^{-M(t-\sigma)} g_{n}(\sigma) \mathrm{d} \sigma .
$$

Using Lebesgue's dominated convergence theorem, we obtain:

$$
\begin{aligned}
\lim _{n \rightarrow \infty} G\left(t+s_{n}\right) & =\int_{-\infty}^{t} e^{-M(t-\sigma)} \lim _{n \rightarrow \infty} g_{n}(\sigma) \mathrm{d} \sigma \text { (By continuity of the semigroup) } \\
& \left.=\int_{-\infty}^{t} e^{-M(t-\sigma)} h(\sigma) \mathrm{d} \sigma \text { (By using }(2.9)\right) \\
& =y(t), \forall t \in \mathbb{R} .
\end{aligned}
$$

We can show by similar way that

$y\left(t-s_{n}\right) \longrightarrow G(t)$ as $n \longrightarrow \infty$,

for each $t \in \mathbb{R}$. This shows that $G \in A A()$.

This means that $y\left(t-s_{n}\right) \longrightarrow G(t)$ as $n \longrightarrow \infty$. Hence, $G(t)$ is an almost automorphic function.

Since $M$ is a continuous transformation, then $v_{1}=M G(t)$ is is almost automorphic. This concludes the proof.

The theorem being proved, we can now begin the resolution of systems of second-order differential equations.

\subsection{Results for Systems of Second-Order Differential Equations}

In the previous subsection, we have studied and obtained the existence and the uniqueness of the almost automorphic solution to a system of first-order differential equations. In this subsection, we study the system:

$A x^{\prime \prime}(t)+B x^{\prime}(t)+C x(t)=f(t)$,

where $A, B, C$ are $m \times m$-square matrices with complex entries such that: $\operatorname{det}(A)=0$, and $f \in A A\left(\mathrm{C}^{2 m}\right)$. 


\subsubsection{Resolution of the problem}

The strategy we use here consists of rewriting Eq. (2.11) as a system of first-order differential equations (See [6] and [1]).

After rewriting (2.11) in the form (2.12), we can now solve it by using the result obtained the in the previous section.

Suppose that $x: \mathbb{R} \longrightarrow \mathbb{C}^{m}$ is a twice differenciable, and set $u=\left(\begin{array}{l}x_{1} \\ x_{2}\end{array}\right)=\left(\begin{array}{c}x \\ x^{\prime}\end{array}\right)$, then it follows:

$$
E u^{\prime}(t)+F u(t)=L(t)
$$

where $E=\left(\begin{array}{cc}B & A \\ I & O\end{array}\right) ; F=\left(\begin{array}{cc}C & O \\ O & -I\end{array}\right)$ and $L=\left(\begin{array}{c}f \\ O\end{array}\right)$.

Where $O$ means the zero matrix and $I$ the identity matrix.

By having the form (2.12), we can use the strategy developped in the above section to find the solution.

It should be noted that $A, B$ and $C$ must be so that $\lambda$ be a simple pole of the resolvent

$R(\lambda, T):=(\lambda I=T)_{-1}^{-1}$, where $T$ a matrix resulting form the transformation of Eq.(2.12) by following the steps of the section 2.2.

Hence, the following theorem:

Théorème 2.3.1. Under previous assumptions, if $\mathbf{f} \in \mathrm{AA}\left(\mathrm{C}^{\mathrm{m}}\right)$, then Eq. (2.11) has a unique solution, and this solution is almost automorphic.

The proof of Theorem 2.3.1 follows along the same lines as that Theorem 2.2.1 and hence omitted.

3. Example

To apply our theoretical results, we consider the following system of differential equations [11]

$$
E u^{\prime}(t)+F u(t)=g(t), t \in \mathbb{R}
$$

where: $m=2$, and $E=\left(\begin{array}{ll}2 & 4 \\ \frac{1}{2} & 1\end{array}\right) \in M(2, \mathbb{C}), F=\left(\begin{array}{ll}0 & 1 \\ 1 & 1\end{array}\right) \in M(2, \mathbb{C})$

and $g(t)=\left(\begin{array}{c}\cos \left(\frac{1}{2+\sin (\sqrt{2} t)+\sin (\sqrt{3} t)}\right) \\ \cos \left(\frac{1}{2+\sin (4 t)+\sin (\sqrt{5} t)}\right)\end{array}\right) \in A A\left(\mathbb{C}^{2}\right)$.

Clearly, $g(t)$ has coefficients which are neither periodic nor almost periodic, but almost automorphic functions.

Let us find $u(t)=\left(\begin{array}{l}u_{1}(t) \\ u_{2}(t)\end{array}\right)$. Now, we have $T=E F^{-1}=\left(\begin{array}{cc}2 & 2 \\ \frac{1}{2} & \frac{1}{2}\end{array}\right)$. Set $F u(t)=v(t)$.

Hence, the system becomes:

$T v^{\prime}(t)+v(t)=g(t) \Longleftrightarrow\left(\begin{array}{ll}2 & 2 \\ \frac{1}{2} & \frac{1}{2}\end{array}\right)\left(\begin{array}{l}v_{1}(t) \\ v_{2}(t)\end{array}\right)^{\prime}+\left(\begin{array}{l}v_{1}(t) \\ v_{2}(t)\end{array}\right)=\left(\begin{array}{c}\cos \left(\frac{1}{2+\sin (\sqrt{2} t)+\sin (\sqrt{3} t)}\right) \\ \cos \left(\frac{1}{2+\sin (4 t)+\sin (\sqrt{5} t)}\right)\end{array}\right)$.

Applying the decomposition of the space, the kernel is

$\mathbb{X}_{2}=N(T)=\operatorname{span}\left(\left(\begin{array}{c}-1 \\ 1\end{array}\right)\right)$.

Let $V_{0}=\left(\begin{array}{l}4 \\ 1\end{array}\right)$ and $V_{1}=\left(\begin{array}{c}-1 \\ 1\end{array}\right)$.

It is easy to see that $R(T)=\operatorname{span}\left(\left(\begin{array}{l}4 \\ 1\end{array}\right)\right)$.

Clearly, $W=\left\{V_{0}, V_{1}\right\}$ is an orthogonal basis for $\mathbb{C}^{2}$. 
The matrix associated to $\mathrm{W}$ is given by

$$
S=\left(\begin{array}{cc}
4 & -1 \\
1 & 1
\end{array}\right)
$$

If we express $T$ in the new basis $W=\left\{V_{0}, V_{1}\right\}$, we get:

$T^{\prime}=\left(\begin{array}{cc}\frac{5}{2} & 0 \\ 0 & 0\end{array}\right)$

Hence, $M^{-1}=\left(\frac{5}{2}\right)$ which yields $M=\left(\frac{2}{5}\right)$

Let us find $g_{1}$ and $g_{2}$, where

$$
\begin{aligned}
P_{\mathrm{X}_{1}} g & =\left(\begin{array}{l}
4 \\
1
\end{array}\right)\left(\left(\begin{array}{l}
4 \\
1
\end{array}\right)\left(\begin{array}{l}
4 \\
1
\end{array}\right)\right)^{-1}\left(\begin{array}{l}
4 \\
1
\end{array}\right)^{T}\left(\begin{array}{c}
\cos \left(\frac{1}{2+\sin (\sqrt{2} t)+\sin (\sqrt{3} t)}\right) \\
\cos \left(\frac{1}{2+\sin (4 t)+\sin (\sqrt{5} t)}\right)
\end{array}\right) \\
& =\left(\begin{array}{ll}
\frac{16}{17} & \frac{4}{17} \\
\frac{4}{17} & \frac{1}{17}
\end{array}\right)\left(\begin{array}{c}
\cos \left(\frac{1}{2+\sin (\sqrt{2} t)+\sin (\sqrt{3} t)}\right) \\
\cos \left(\frac{1}{2+\sin (4 t)+\sin (\sqrt{5} t)}\right)
\end{array}\right) \\
& =\left(\begin{array}{c}
\frac{16}{17} \cos \left(\frac{1}{2+\sin (\sqrt{2} t)+\sin (\sqrt{3} t)}\right)+\frac{4}{17} \cos \left(\frac{1}{2+\sin (4 t)+\sin (\sqrt{5} t)}\right) \\
\frac{4}{17} \cos \left(\frac{1}{2+\sin (\sqrt{2} t)+\sin (\sqrt{3} t)}\right)+\frac{1}{17} \cos \left(\frac{1}{2+\sin (4 t)+\sin (\sqrt{5} t)}\right)
\end{array}\right) \\
& =\left(\begin{array}{l}
\frac{16}{17} \cos \left(\frac{1}{2+\sin (\sqrt{2} t)+\sin (\sqrt{3} t)}\right) \\
\frac{4}{17} \cos \left(\frac{1}{2+\sin (\sqrt{2} t)+\sin (\sqrt{3} t)}\right)
\end{array}\right)+\left(\begin{array}{c}
\frac{4}{17} \cos \left(\frac{1+\sin (4 t)+\sin (\sqrt{5} t)}{2+\sin (\sqrt{5} t)}\right) \\
\frac{1}{17} \cos \left(\frac{1}{2+\sin (4 t)+\sin }\right)
\end{array}\right) \\
& =\frac{4}{17} \cos \left(\frac{1}{2+\sin (\sqrt{2} t)+\sin (\sqrt{3} t)}\right)\left(\begin{array}{l}
4 \\
1
\end{array}\right)+\frac{1}{17} \cos \left(\frac{1}{2+\sin (4 t)+\sin (\sqrt{5} t)}\right)\left(\begin{array}{l}
4 \\
1
\end{array}\right) \\
& =\left[\frac{4}{17} \cos \left(\frac{1}{2+\sin (\sqrt{2} t)+\sin (\sqrt{3} t)}\right)+\frac{1}{17} \cos \left(\frac{1}{2+\sin (4 t)+\sin (\sqrt{5} t)}\right)\right]\left(\begin{array}{l}
4 \\
1
\end{array}\right) \\
& =g_{1} V_{0}
\end{aligned}
$$

with $g_{1}=\frac{4}{17} \cos \left(\frac{1}{2+\sin (\sqrt{2} t)+\sin (\sqrt{3} t)}\right)+\frac{1}{17} \cos \left(\frac{1}{2+\sin (4 t)+\sin (\sqrt{5} t)}\right)$ and

$$
\begin{aligned}
P_{\mathrm{X}_{2}} g & =\left(\begin{array}{c}
-1 \\
1
\end{array}\right)\left(\left(\begin{array}{c}
-1 \\
1
\end{array}\right)^{T}\left(\begin{array}{c}
-1 \\
1
\end{array}\right)\right)^{-1}\left(\begin{array}{c}
-1 \\
1
\end{array}\right){ }^{T}\left(\begin{array}{c}
\cos \left(\frac{1}{2+\sin (\sqrt{2} t)+\sin (\sqrt{3} t)}\right) \\
\cos \left(\frac{1}{2+\sin (4 t)+\sin (\sqrt{5} t)}\right)
\end{array}\right) \\
& =\left(\begin{array}{cc}
\frac{1}{2} & -\frac{1}{2} \\
-\frac{1}{2} & \frac{1}{2}
\end{array}\right)\left(\begin{array}{c}
\cos \left(\frac{1}{2+\sin (\sqrt{2} t)+\sin (\sqrt{3} t)}\right) \\
\cos \left(\frac{1}{2+\sin (4 t)+\sin (\sqrt{5} t)}\right)
\end{array}\right) \\
& =\left(\begin{array}{c}
\frac{1}{2} \cos \left(\frac{1}{2+\sin (\sqrt{2} t)+\sin (\sqrt{3} t)}\right)-\frac{1}{2} \cos \left(\frac{1}{2+\sin (4 t)+\sin (\sqrt{5} t)}\right) \\
-\frac{1}{2} \cos \left(\frac{1}{2+\sin (\sqrt{2} t)+\sin (\sqrt{3} t)}\right)+\frac{1}{2} \cos \left(\frac{1}{2+\sin (4 t)+\sin (\sqrt{5} t)}\right)
\end{array}\right) \\
& =\left(\begin{array}{c}
\frac{1}{2} \cos \left(\frac{1}{2+\sin (\sqrt{2} t)+\sin (\sqrt{3} t)}\right) \\
-\frac{1}{2} \cos \left(\frac{1}{2+\sin (\sqrt{2} t)+\sin (\sqrt{3} t)}\right)
\end{array}\right)-\left(\begin{array}{c}
\frac{1}{2} \cos \left(\frac{1}{2+\sin (4 t)+\sin (\sqrt{5} t)}\right) \\
-\frac{1}{2} \cos \left(\frac{1}{2+\sin (4 t)+\sin (\sqrt{5} t)}\right)
\end{array}\right) \\
= & \frac{1}{2} \cos \left(\frac{1}{2+\sin (\sqrt{2} t)+\sin (\sqrt{3} t)}\right)\left(\begin{array}{c}
1 \\
-1
\end{array}\right)-\frac{1}{2} \cos \left(\frac{1}{2+\sin (4 t)+\sin (\sqrt{5} t)}\right)\left(\begin{array}{c}
1 \\
-1
\end{array}\right) \\
= & {\left[\frac{1}{2} \cos \left(\frac{1}{2+\sin (\sqrt{2} t)+\sin (\sqrt{3} t)}\right)-\frac{1}{2} \cos \left(\frac{1}{2+\sin (4 t)+\sin (\sqrt{5} t)}\right)\right]\left(\begin{array}{c}
1 \\
-1
\end{array}\right) } \\
P_{\mathrm{X}_{2}} g & =g_{2}(t) V_{1} \\
\text { with } g_{2}(t)= & \frac{1}{2} \cos \left(\frac{1}{2+\sin (\sqrt{2} t)+\sin (\sqrt{3} t)}\right)-\frac{1}{2} \cos \left(\frac{1}{2+\sin (4 t)+\sin (\sqrt{5} t)}\right) .
\end{aligned}
$$


Now, the system $\left(\begin{array}{ll}2 & 2 \\ \frac{1}{2} & \frac{1}{2}\end{array}\right)\left(\begin{array}{l}v_{1}(t) \\ v_{2}(t)\end{array}\right)^{\prime}+\left(\begin{array}{l}v_{1}(t) \\ v_{2}(t)\end{array}\right)=\left(\begin{array}{c}\cos \left(\frac{1}{2+\sin (\sqrt{2} t)+\sin (\sqrt{3} t)}\right) \\ \cos \left(\frac{1}{2+\sin (\sqrt{2} t)+\sin (\sqrt{3} t)}\right)\end{array}\right)$ can be written in the following form:

$$
\left\{\begin{aligned}
M^{-1} v_{1}^{\prime}(t)+v_{1}(t) & =g_{1}(t) \\
v_{2}(t) & =g_{2}(t)
\end{aligned}\right.
$$

We have

$$
\left\{\begin{aligned}
\frac{5}{2} v_{1}^{\prime}(t)+v_{1}(t) & =\frac{1}{17}\left[4 \cos \left(\frac{1}{2+\sin (\sqrt{2} t)+\sin (\sqrt{3} t)}\right)+\cos \left(\frac{1}{2+\sin (4 t)+\sin (\sqrt{5} t)}\right)\right], \\
v_{2}(t) & =\frac{1}{2}\left[\cos \left(\frac{1}{2+\sin (\sqrt{2} t)+\sin (\sqrt{3} t)}\right)-\cos \left(\frac{1}{2+\sin (4 t)+\sin (\sqrt{5} t)}\right)\right] .
\end{aligned}\right.
$$

Applying Theorem 3.2.2, the unique almost automorphic solution of the above system is given by

$$
\begin{aligned}
v_{1}(t) & =e^{-M(t-\sigma)} v_{1}(\sigma)+M \int_{\sigma}^{t} e^{-M(t-s)} g_{1}(s) \mathrm{d} s \\
& =e^{-\frac{2}{5}(t-\sigma)} v_{1}(\sigma)+\frac{2}{85} \int_{\sigma}^{t} e^{-\frac{2}{5}(t-s)}\left[4 \cos \left(\frac{1}{2+\sin (\sqrt{2} s)+\sin (\sqrt{3} s)}\right)+\cos \left(\frac{1}{2+\sin (4 s)+\sin (\sqrt{5} s)}\right)\right] \mathrm{d} s
\end{aligned}
$$

and

$$
v_{2}(t)=\frac{1}{2}\left[\cos \left(\frac{1}{2+\sin (\sqrt{2} t)+\sin (\sqrt{3} t)}\right)-\cos \left(\frac{1}{2+\sin (4 t)+\sin (\sqrt{5} t)}\right)\right] .
$$

Since $F u(t)=v(t)$ and $F^{-1}=\left(\begin{array}{cc}-1 & 1 \\ 1 & 0\end{array}\right)$ we have

$$
u(t)=F^{-1} v(t) \Longleftrightarrow u(t)=\left(\begin{array}{cc}
-1 & 1 \\
1 & 0
\end{array}\right)\left(\begin{array}{l}
v_{1}(t) \\
v_{2}(t)
\end{array}\right) .
$$

Since $F^{-1}$ is a continuous transformation and $v(t)$ is an almost automorphic function, the unique solution of (3.1) $u(t)=F^{-1} v(t)$ is almost automorphic. Hence, $u(t)$ is given by

$$
u(t)=\left(\begin{array}{c}
v_{2}(t)-v_{1}(t) \\
v_{1}(t)
\end{array}\right) .
$$

\section{Conclusion}

In this paper, we studied and found the existence and uniqueness of almost automorphic solutions to some singular systems differential equations with constant coefficients. The system we have studied is a nonhomogeneous system with almost automorphic forcing term.

So, there are still some problems that are not solved yet. As future work, we suggest the following:

1. The study of nonautonomous cases, that is,

$E(t) u^{\mathrm{J}}(t)+F(t) u(t)=g(t), t \in \mathrm{R}$

where $E(t), F(t) \in M(m, \mathrm{C})$ and $f \in A A\left(\mathrm{C}^{m}\right)$ and

$A(t) u^{t t}(t)+B(t) u^{t}+C(t) u(t)=f(t), t \in \mathrm{R}$

where $A(t), B(t), C(t) \in M(m, \mathrm{C})$ and $f \in A A\left(\mathrm{C}^{m}\right)$.

2. The study of higher-order case, that is

$A_{m} x^{(m)}(t)+A_{m-1} x^{(m-1)}+\ldots+A_{1} x^{\mathrm{J}}(t)+A_{0} x(t)=f(t), t \in \mathrm{R}$,

where $A_{i} \in M(n, \mathrm{C})$ and $m \geq 3$.

\section{References}

[1] M. Arianmughare and T. Diagana. Existence of Almost Periodic Solutions to some Singular Differential Equations. Nonlinear Dyn. Syst. Theory, 13(1):1-12,2013. 
[2] S. Bochner. A new approach to almost periodicity. Proc. Nat. Acad. Sci. U.S.A., 48(12):2039- 2043, 1962.

[3] H. Bohr. Zur theorie der fast periodischen functionen. Acta Mathematica, 45:29-197, 1925.

[4] H. Bohr. Almost Periodic Functions. Chelsea Publishing Company. Springer, Berlin, 1947.

[5] D. Bugajewski and T. Diagana. Almost Automorphy of the Convolution Operator and Application To Differential and Functional Differential Equations. Nonlinear Studies, 13(2):129- 140, 2006.

[6] S.L. Campbel. Singular systems of differential equations. Pitman Advanced Publishing Pro- gram. Research Notes in Mathematics, London, 1980.

[7] C. Corduneanu. Almost Periodic Functions. Chealsea Publishing Company, New York, second english edition, 1989.

[8] T. Diagana. Almost Automorphic Type and Almost Periodic Type Functions in Abstract Spaces. Springer International Publishing. Springer, Berlin, 2013.

[9] Diagana and M.M. Mbaye. Square-mean almost periodic periodic solutions to some singular stohastic differential equations. Appl. Math. Lett., 54:48-53, 2016.

[10] A. Favini, V. Maksimov, and L. Pandolfi. A deconvolution problem related to a singular system. J. Math. Anal. Appl., (292):60-72,2004.

[11] G. L. Kamingu. Existence of Almost Automorphic Solutions to some Singular Systems of Dif- ferential Equations. Master II Thesis in Mathematical Sciences. African Institute for Mathe- matical Sciences (AIMS)/Senegal, 2017.

[12] G. M. N'Guérékata. Almost Automorphic and Almost Periodic Functions in Abstract Spaces. Springer Science+Business Media. Springer, New York, 2001.

[13] W.A Veech. Almost automorphic functions. Proc. Nat. Acad. Sci. U.S.A., 49(4):462-464, 1963.

[14] Y. Yi and W. Shen. Almost Automorphic and Almost Periodic Dynamics in Skew-product Semiflows, volume 647 of Memoirs of the American Mathematical Society. Amer. Math. Soc., 1998.

[15] M. Zaki. Almost auromorphic integrals of almost automorphic functions. Canad. Math. Bull., 15(3):433-436, 1972.

Citation: Gradi KAMINGU LUBWELE (2019). Study of Existence of Almost Automorphic Solutions to Some Singular Systems of Differential Equations. International Journal of Scientific and Innovative Mathematical Research (IJSIMR), 7(10), pp. 5-20. http://dx.doi.org/ 10.20431/2347 -3142.0710002

Copyright: () 2019 Authors, this is an open-access article distributed under the terms of the Creative Commons Attribution License, which permits unrestricted use, distribution, and reproduction in any medium, provided the original author and source are credited. 